\title{
El papel de la confianza en proyectos de innovación colaborativa. Propuesta teórico-metodológica
}

\section{The role of trust in collaborative innovation projects. \\ A theoretical-methodological proposal}

\author{
Gabriela Pino García* \\ Ismael Aguilar Barajas* \\ Edgardo Arturo Ayala Gaytán*
}

\begin{abstract}
This paper addresses the role of trust in the generation of collaborative innovation. The research methodologies, antecedents, temporality, and conceptualization of trust are analyzed. The foregoing is linked to its dimensions and forms of operationalization that have been used in different contexts to understand collaborative innovation. A theoretical-methodological model is developed to explain the construction of trust in innovation projects, as a complex and expensive process. The model can be applied in different areas, including territorial, for example, in the evaluation of regional innovation systems.
\end{abstract}

Keywords: trust, collaborative innovation, innovation projects, regional innovation systems.

\section{Resumen}

Este artículo aborda el papel de la confianza en la generación de innovación colaborativa. Se analizan las metodologías de investigación, antecedentes, temporalidad y conceptualización de la confianza. Lo anterior se enlaza con sus dimensiones y formas de operacionalización que se han utilizado en diferentes contextos para entender la innovación colaborativa. Se desarrolla un modelo teórico-metodológico para explicar la construcción de la confianza en proyectos de innovación, como un proceso complejo y costoso. El modelo puede ser aplicado en distintos ámbitos, incluido el territorial, por ejemplo, en la evaluación de los sistemas de innovación regionales.

Palabras clave: confianza, innovación colaborativa, proyectos de innovación, sistemas de innovación regional.

*Tecnológico de Monterrey, Campus Monterrey, correos-e: gabypino@gmail.com, iaguilar@ itesm.mx, edgardo@itesm.mx 


\section{Introducción}

Cuando se habla de innovación se hace referencia a la mejora o desarrollo de un nuevo producto, servicio, proceso, estrategia de mercadotecnia o método organizacional, el cual es comercializado o implementado internamente (OECD y Eurostat, 2005). La innovación es un proceso continuo de exploración y explotación, abarca desde la búsqueda y experimentación hasta el descubrimiento, la manufactura y su comercialización (van Hemert et al., 2013). El resultado de este proceso debe generar algún tipo de valor para todos los actores involucrados, ya sea en el ámbito económico, de conocimiento, de capital social, entre otros. La generación de innovación es un proceso complejo y dinámico que depende de la alineación de elementos. Entre los principales determinantes de la innovación se encuentran: el entorno de la organización y la disponibilidad de recursos económicos, la cultura y la estructura organizacional, los rasgos del equipo y la cooperación efectiva. La interacción de estos determinantes dentro de un ambiente de confianza facilita la innovación colaborativa. ${ }^{1}$ Estos determinantes presentan variaciones nacionales y subnacionales. Es tanta la variación de estos determinantes entre cada región que a pesar de que varios de ellos estén presentes en ese ambiente para la innovación, no hay garantía alguna de que se genere innovación colaborativa (Salas et al., 2003; O’Mara, 2010).

En la mayoría de los casos, la innovación implica colaboración y para que ésta sea efectiva es indispensable contar con un equipo de innovación, el cual puede estar conformado por representantes de la misma organización o de distintas organizaciones, quienes se enfocan en llegar a un objetivo específico y concluir un proyecto. La motivación, la buena actitud, el sentido de urgencia y el apoyo emocional y financiero son necesarios para que los integrantes del equipo responsable puedan ejecutar un proyecto adecuadamente. Cuando el equipo está conformado por representantes de diferentes organizaciones - la cooperación efectiva, los contratos claros, la comunicación abierta, la transparencia y el sentido de responsabilidad - son aspectos que impactan directamente en el desempeño y los resultados finales. Aunado a esto, es necesario nutrir el capital social, complementar los conocimientos del socio, compartir por objetivos e intereses, sentir interdependencia y seguridad y, sobre todo, fundamentar la relación en la confianza mutua. Inclusive, para que la

\footnotetext{
${ }^{1}$ Las categorías de los determinantes de la innovación y sus respectivas descripciones están basadas en las siguientes investigaciones: Salas et al. (2003); Bastic y Leskovar-Spacapan (2006); Rampersad et al. (2010); Arata et al. (2011); Fritsch y Slavtchev (2011); Sie et al. (2014), y Lemus Delgado et al. (2015).
} 
relación inicie es necesario cierto grado de confianza. De aquí la relevancia de los contextos iniciales.

La confianza es un tema central desde el inicio de cualquier relación comercial o de innovación colaborativa entre organizaciones (Brattström et al., 2015). La confianza permite el intercambio de información, conocimiento e ideas; así como la coordinación de las actividades en ambientes complejos y de incertidumbre. En las relaciones interorganizacionales, la confianza disminuye la sensación de incertidumbre y riesgo en la toma de decisiones. Las decisiones y los tratos que se realicen pueden basarse en la palabra del socio o pueden venir acompañadas de contratos o instrumentos que la sustenten. Dichos instrumentos pueden complementar la confianza en la relación o simplemente ayudar a formalizar procedimientos, roles, obligaciones, objetivos y estructuras antes de comenzar un proyecto de innovación colaborativa.

Este artículo contribuye a la literatura sobre el papel de la confianza en los procesos de innovación colaborativa de tres formas. La primera tiene el propósito de analizar cuáles han sido las metodologías y técnicas que se han utilizado para el estudio de la confianza en contextos de innovación. Para ello se realizó una búsqueda de artículos sobre confianza e innovación, publicados entre 2000 y 2017, ${ }^{2}$ utilizando diferentes bases de datos de revistas internacionales. La segunda contribución es presentar una tipología que facilita la identificación de las distintas variables que componen ese estado inicial de confianza, a través del análisis de los antecedentes que permiten el establecimiento de una confianza inicial para el comienzo de proyectos de innovación. Finalmente, la tercera aportación de este trabajo es proveer de una operacionalización de la confianza y las dimensiones de la misma. Se propone un modelo que combina metodologías y toma en consideración el contexto de innovación y la temporalidad de la confianza, con la finalidad de reducir la brecha entre la conceptualización y las formas de operacionalizar los constructos. Se concluye que la dimensión espacial ofrece avenidas promisorias para probar empíricamente este modelo.

\footnotetext{
${ }^{2}$ Anteriormente ya se han realizado investigaciones en las que se analizan artículos que estudian el tema de la confianza en contextos de innovación. Esto ha sido con el objetivo de identificar las metodologías utilizadas para el análisis, sus dimensiones, sus formas de conceptualización y operacionalización. Un claro ejemplo ha sido la investigación realizada por Seppänen et al. (2007), en la que se consideraron publicaciones de 1990-2003, sin discriminar el tipo de relación entre los socios el cual ha resultado ser un elemento importante para el entendimiento del fenómeno. Las investigaciones más recientes sugieren un cambio en la manera en que se estudia este fenómeno, lo cual enmarca un nuevo paradigma que ha venido evolucionando a partir del 2000. Vale la pena su revisión.
} 


\section{Metodologías utilizadas para el estudio de la confianza en contextos de innovación}

Para la revisión de los estudios de confianza en el contexto de innovación se utilizaron diferentes bases de datos de revistas internacionales como ScienceDirect, EbscoHost, así como publicaciones en Academia y Google Scholar. De los 77 documentos encontrados, se seleccionaron 13 investigaciones empíricas con muestras de estudio en las que aparecen empresas, universidades, centros de investigación e instituciones de distintos países. Se dejaron fuera las investigaciones en ambientes controlados, con estudiantes o en escenarios hipotéticos. Se identificó que la mercadotecnia, los estudios regionales, la administración, la psicología, la sociología, la economía y los negocios son algunas de las diferentes aproximaciones teóricas que han abonado al entendimiento de este fenómeno. Se omitieron aquellos estudios en los que la relación social se reducía a negociaciones para la compra-venta; en los que se vinculaba el concepto de confianza con la adopción de innovación por parte de los consumidores; o cualquier otro tipo de relación entre las organizaciones y los consumidores. El enfoque se sostuvo en los estudios que relacionaban la confianza con la generación de innovación o la transferencia de conocimiento.

Uno de los principios básicos para poder comprender un fenómeno es definirlo o conceptualizarlo. En el caso de la confianza, su conceptualización ha sido uno de los retos más importantes. Difícilmente se puede encontrar una definición de la confianza que se pueda aplicar de manera universal. En ocasiones, al ser un elemento presente en todos los ámbitos de la vida, se da por sentado que los sujetos de estudio e inclusive los mismos lectores conocen de antemano su definición. Sin embargo, como se explicó, al ser un concepto complejo, dinámico y adaptable, según el contexto en el que se observa, es indispensable establecer una definición como punto de referencia que sirva de apoyo para obtener resultados más certeros al momento de implementar metodologías de investigación. A continuación, se muestran algunas de las definiciones presentadas en los artículos que se seleccionaron para el presente análisis:

- Un principio informal organizativo que implica tener expectativas positivas en relación con la benevolencia e integridad del otro. (Mayer et al., 1995, citado en Brattström et al., 2015: 3)

- Un estado psicológico que implica aceptar la vulnerabilidad basándose en las expectativas positivas sobre las intenciones o el comportamiento de otros. (Rousseau et al., 1998)

- Un estado que implica expectativas positivas confiadas sobre los motivos de otro con respecto a uno mismo en situaciones que 
implican riesgo. (Boon y Holmes, 1991, citado en Lewicki y Bunker, 1995: 139)

- Un bien condicionado esencial en la formulación de procesos estratégicos. (Molina-Morales et al., 2011)

A pesar de que no todas las definiciones de confianza son iguales, la mayoría de los investigadores coinciden en que posee las siguientes características: ${ }^{3}$ surge en las relaciones sociales, se constituye con base en las expectativas sobre las intenciones o el comportamiento de otros, permite la vulnerabilidad y riesgo en cualquier relación, y cambia en el tiempo. Con base en lo anterior, se puede elegir la metodología y los métodos que permitan comprender su funcionamiento y el impacto que tiene en variables como el éxito, el desempeño y la innovación, entre otras.

La metodología elegida puede tener un enfoque cualitativo, cuantitativo o mixto, los cuales tienen sus respectivas ventajas y desventajas. Por un lado, la dimensión cuantitativa le da importancia a lo objetivo y está relacionada con la cantidad o magnitud. Por el otro lado, la dimensión cualitativa proporciona la información para comprender cómo y de qué está constituido un determinado fenómeno, así como los elementos que lo integran. Ambos enfoques metodológicos permiten explicar los fenómenos desde diferentes perspectivas; pero ninguno de los dos se puede considerar por sí solo como una solución para los problemas que se plantean en las investigaciones sociales. No obstante, al combinarlos existe una gran posibilidad de neutralizar las fallas de un enfoque y fortalecer los beneficios del otro. Las metodologías con enfoques mixtos proporcionan información para obtener mejores resultados, incrementar la validez y credibilidad de la investigación, y explicar de manera más amplia y profunda el fenómeno de estudio (Hussein, 2009).

Es por eso que, para comprender la relación entre confianza e innovación colaborativa es indispensable también profundizar y cuestionar la manera en la que ha sido estudiada. Esto incluye el enfoque metodológico utilizado, el tipo de confianza que se estudia (interpersonal o interorganizacional) y el país o región a la que pertenece la muestra de estudio. En las investigaciones seleccionadas, se identificaron los tres tipos de enfoques metodológicos (tabla 1): cuantitativa, cualitativa y mixta. A pesar de que existen diversos niveles de análisis de confianza, se vislumbra el interés de los autores por entender la confianza interorganizacional e interpersonal,

\footnotetext{
${ }^{3}$ En varias de las investigaciones se asume que el lector conoce de antemano la definición de confianza. Se consideraron como referencia los artículos que conceptualizan la confianza como parte de su metodología entre los cuales se encuentran: Lewicki y Benedict Bunker (1995), Ruppel y Harrington (2000), Fang et al. (2008), Maurer (2010), Molina-Morales et al. (2011), BabiliuteJuceviciene et al. (2013), Brattström et al. (2015), y Brinkhoff et al. (2015).
} 
principalmente. La confianza interorganizacional (CIO) se refiere a la colaboración entre dos entidades diferentes; en cambio, la interpersonal (CIP) apunta hacia las relaciones dentro de la misma organización o entre los representantes de las organizaciones que participan en el proyecto. Como complemento, se realizó una distinción entre los países a los que pertenece la muestra de estudio, ya que la confianza generalizada del país o región en la que se realiza la investigación, puede tener injerencia en el nivel de confianza inicial de cualquier relación debido a la cultura, las costumbres y las tradiciones relacionadas con el contexto.

Diez de los 13 artículos de investigación seleccionados optaron por un enfoque metodológico cuantitativo, con información recabada mediante cuestionarios. En un tercio de estos artículos se analizó la confianza interpersonal (CIP), y en el resto, la confianza interorganizacional (CIO). ${ }^{4}$ Las muestras de estudio provinieron de Suecia, Alemania, España, China y Taiwán. ${ }^{5}$ Se utilizaron escalas de Likert para medir los constructos y posteriormente aplicar métodos de análisis, tales como regresiones, correlaciones, factores, componentes principales y modelos de ecuaciones estructurales. Por otro lado, en tres estudios se adoptó una metodología cualitativa con información recopilada por medio de entrevistas o algún tipo de etnografía, con muestras de Inglaterra, Estados Unidos de América y Taiwán. Un tercio de ellos se enfocaron en la confianza interpersonal del equipo de innovación. Esta muestra de artículos sugiere una tendencia hacia el análisis cuantitativo en el estudio de la confianza.

Inesperadamente, sólo una de las 13 investigaciones eligió una metodología mixta. En ese estudio, los investigadores realizaron entrevistas para probar el cuestionario. Lamentablemente, las preguntas de las entrevistas estaban enfocadas en el entendimiento por parte del entrevistado sobre cada uno de los conceptos del cuestionario y a detectar algunos errores de redacción. Es decir, en ese caso, la metodología mixta fungió de apoyo para cuestiones de forma más que para el entendimiento del fenómeno. Dicho acercamiento pudo haber sido aprovechado para la construcción propia del cuestionario, la identificación de los antecedentes de la confianza, las principales variables del contexto, la percepción sobre la predisposición a confiar y colaborar u otro tipo de aspectos más relevantes.

A pesar de que la selección de investigaciones es una muestra del avance en la conceptualización y la validez de las escalas de medición de la confianza, existen fisuras en su comprensión. La tendencia positivista en el estudio de este fenómeno ha limitado su explicación a la transformación de conceptos cualitativos en constructos cuantitativos para su posterior

\footnotetext{
${ }^{4}$ Solamente en el artículo de Fang et al. (2008) se analizaron ambos tipos de confianza —interpersonal e interorganizacional- por separado.

${ }^{5}$ Los autores consideran a China y Taiwán como regiones independientes una de la otra.
} 


\section{Tabla 1}

\section{Enfoques metodológicos para el estudio de la confianza}

\begin{tabular}{|c|c|c|c|}
\hline $\begin{array}{l}\text { Enfoque } \\
\text { metodológico }\end{array}$ & $\begin{array}{l}\text { Tipo de } \\
\text { confianza }\end{array}$ & Aproximación teórica & $\begin{array}{c}\text { Autores. } \\
\text { (Región de la muestra de estudio) }{ }^{1}\end{array}$ \\
\hline \multirow[t]{2}{*}{$\begin{array}{l}\text { Cuantitativo: } \\
\text { cuestionario }\end{array}$} & $\begin{array}{l}\mathrm{CIP}^{2} \\
\text { equipo de } \\
\text { innovación }\end{array}$ & $\begin{array}{l}\text { Administración, } \\
\text { mercadotecnia y } \\
\text { psicología social }\end{array}$ & $\begin{array}{l}\text { Fang et al., } 2008 \text { (China); Hu } \\
\text { et al., } 2012 \text { (Taiwán); Brattström } \\
\text { et al., } 2015 \text { (Suecia) }\end{array}$ \\
\hline & $\mathrm{CIO}^{3}$ & $\begin{array}{l}\text { Administración, } \\
\text { ciencias sociales, } \\
\text { economía, psicolo- } \\
\text { gía social, mercado- } \\
\text { tecnia }\end{array}$ & $\begin{array}{l}\text { Ruppel y Harrington, } 2000 \\
\text { (n.d.); Wei et al., } 2006 \text { (Zhe- } \\
\text { jiang, China); Fang et al., } 2008 \\
\text { (China); Maurer, } 2010 \text { (Alema- } \\
\text { nia); Molina-Morales et al., } \\
2011 \text { (Valencia, España); } \\
\text { Babiliute-Juceviciene et al., } 2013 \\
\text { (n.d.); Brinkhoff et al., } 2015 \\
\text { (Europa) }\end{array}$ \\
\hline \multirow[t]{2}{*}{$\begin{array}{l}\text { Cualitativo: } \\
\text { etnografía o } \\
\text { entrevistas }\end{array}$} & $\begin{array}{l}\text { CIP, } \\
\text { equipo de } \\
\text { innovación }\end{array}$ & Sociología & $\begin{array}{l}\text { Jirotka et al., } 2005 \text { (Inglaterra, } \\
\text { Reino Unido) }\end{array}$ \\
\hline & $\mathrm{CIO}$ & $\begin{array}{l}\text { Administración y } \\
\text { filosofía de la } \\
\text { ingeniería }\end{array}$ & $\begin{array}{l}\text { Wei et al., } 2008 \text { (Taiwán); } \\
\text { Wilcox, } 2016 \text { (Estados Unidos } \\
\text { de América) }\end{array}$ \\
\hline $\begin{array}{l}\text { Mixto: } \\
\text { entrevista y } \\
\text { cuestionario }\end{array}$ & $\mathrm{CIO}$ & Administración & Williams y Du, 2014 (China) \\
\hline
\end{tabular}

${ }^{1}$ Resultados de la encuesta realizada por World Values Survey (WVS, 2015) demuestran que las actitudes hacia la confianza interpersonal generalizada varían según el contexto. A continuación, se presenta el porcentaje de personas por país que estuvieron de acuerdo con el enunciado se puede confiar en la mayoría de las personas: 63.9, China; 63.7, Suecia; 42, Alemania; 38.1, Estados Unidos de América; 29.9, Reino Unido - dato 2009-; 29.6, Taiwán, y 19, Espańa. Algunos de los artículos analizados en el presente escrito no proporcionaron el país de la muestra de estudio a pesar de su importancia para el entendimiento del fenómeno. Cabe aclarar que el nivel de confianza generalizada es un antecedente que construye, en conjunto con otros elementos, la confianza inicial de cualquier relación.

${ }^{2}$ Confianza interpersonal (CIP)

${ }^{3}$ Confianza interorganizacional (CIO)

Fuente: Elaboración propia con base en los autores citados.

evaluación y ratificación. Lo que se observa es una relación social con un mayor número de matices que va del uno al siete; con una naturaleza variable y volátil; y con una serie de motivos, eventos y antecedentes que hasta la fecha no han llegado a ser protagonistas en las metodologías de estudio. No hay que olvidar que la confianza se ve afectada por las emociones y sentimientos como el miedo, la sospecha, la amistad, la esperanza, la desesperación y demás cuestiones humanas que difícilmente se pueden 
detectar en un cuestionario (Nooteboom, 2006). Es por ello que falta mucho por explicar y entender en este campo. La metodología mixta pudiera ser una manera de complementar el conocimiento que ya se tiene y continuar con el estudio de la confianza en sus diferentes contextos.

\section{Antecedentes de la confianza para el desarrollo de proyectos de innovación colaborativa}

Para estudiar las relaciones en proyectos de innovación, como en cualquier otro tipo de proyecto, es necesario considerar que estos tienen un inicio, un desarrollo y un final. Las relaciones evolucionan gracias a la interacción constante, y a menudo hay un periodo de prueba antes de que maduren o se establezcan. En el ámbito organizacional, la confianza es la primicia de toda relación; de no ser así, se perdería la oportunidad de iniciar una negociación (Dwiveldi et al., 2003). La etapa inicial de la confianza se conforma con los antecedentes percibidos o estudiados sobre el comportamiento, la reputación, las capacidades y la historia de cada una de las partes. Este periodo puede considerarse de prueba y puede tener lugar durante las negociaciones iniciales del proyecto en el que se colaborará. Los antecedentes se ubican en un momento previo a tomar la decisión de iniciar una relación de confianza; por lo tanto, pueden ser medidos tomando en cuenta un momento determinado de la relación. Este primer resultado de medición corresponde al nivel de confianza inicial. A pesar de la importancia que tienen los antecedentes sobre ese estado inicial, los autores de los artículos analizados en el presente escrito no suelen hacer énfasis en esto.

Los antecedentes determinan la postura de una organización o persona a partir de conjeturas entre las que se encuentran las siguientes: si es seguro confiar o no, qué tanto confiar y los riesgos, implicaciones e incertidumbre que esto conlleva. Estas precondiciones pueden ser de diversa índole y cambiar según el tipo de proyecto. Es por esto que a continuación se propone una tipología general de antecedentes de la confianza para proyectos de innovación, basada en la revisión de literatura. Se pudieron distinguir ocho categorías de antecedentes: ${ }^{6} 1$. contexto; 2 . políticas, normas e instituciones; 3 . redes; 4. relación interorganizacional; 5 . organizacionales; 6. proyecto de innovación; 7. equipo de innovación y 8. características personales.

${ }^{6}$ Para la categorización de los antecedentes de la confianza se analizaron los siguientes artículos con la finalidad de complementar la bibliografía seleccionada: Ahuja (2000), Antoine (2007), Boughanbouz et al., (2013); Gulati et al. (2000); Hummels y Roosendaal (2001); Ingstrup (2013); Kern y Willcocks (2002); López-Jiménez (2010); Maurer (2010); y Rousseau et al. (1998). 
Los antecedentes vinculados al contexto externo de las organizaciones tienen injerencia en el nivel de confianza inicial generalizado y, en múltiples ocasiones, impactan directamente en el nivel de confianza inicial de un proyecto colaborativo. Los antecedentes contextuales comprenden desde las prácticas culturales, los valores, las creencias y las tradiciones de la región; hasta las particularidades del ambiente al que pertenece la organización. Además, se toman en cuenta las características del sector productivo, la existencia de facilitadores y la arbitrariedad para la resolución de conflictos por un tercer actor. La segunda categoría considera aspectos observados desde la perspectiva externa e interna de las organizaciones. Enmarca principalmente las políticas, las normas y los elementos relacionados con la calidad institucional. La perspectiva externa se refiere a las facilidades o impedimentos del Gobierno, instituciones públicas u otro tipo de estructuras jerárquicas, tales como corporativos o agrupamientos empresariales. En cambio, el enfoque interno está relacionado con las políticas de innovación y gestión de recursos de cada organización; la formalidad de sus procesos; $y$, principalmente, al uso y especificidad de los instrumentos que generan para concretar acuerdos.

La pertenencia a las redes empresariales, de emprendimiento o innovación es un elemento que influye en la construcción de la confianza en las relaciones interorganizacionales. Esta tercera categoría de antecedentes de la confianza puede tener influencia positiva o negativa. Por un lado, a los socios prospecto se les facilita obtener información sobre la reputación de la organización evaluada, así como del nivel de colaboración y participación que tienen en la red. Por otro lado, el grado de reputación, valores y cultura de la misma red pueden recaer sobre la percepción que se tiene del candidato a socio; y según sea el caso, perjudicar o beneficiar su imagen. En el caso en que la organización prospecto no pertenezca a ningún tipo de red, si el proyecto es ejecutado por dos o más organizaciones se toman en cuenta varias cuestiones que corresponden a la relación interoganizacional, tales como: las experiencias previas; la confianza entre los representantes del proyecto; la frecuencia de interacción; la familiaridad biográfica; la proximidad física o sectorial; la alineación y congruencia de ideas, intereses y objetivos; el grado de transferencia de conocimiento entre ellos; la semejanza entre culturas organizacionales; la simetría de poder, decisión e inversión de recursos; la especificidad y formalidad de los acuerdos; el grado de monitoreo foráneo o local y la calidad de la comunicación. Dichos elementos, en conjunto con los de la categoría de redes, son los que permiten distinguir entre la medición del grado de confianza interpersonal o interorganizacional.

Las características propias de cada organización influyen a su vez en los antecedentes que construyen la confianza inicial de toda relación-innovación 
colaborativa. Entre los distintos elementos se pueden mencionar principalmente los siguientes: antigüedad, tipo de industria a la que pertenecen, competencia profesional, percepción que tienen los representantes sobre la vulnerabilidad de la organización, prácticas éticas, reputación, factores socio-morales de la cultura organizacional, comunicación entre empleados, normas y políticas; interés por la innovación; consistencia en los acuerdos, es decir, su desempeño en proyectos anteriores y sus planes a futuro. En la sexta categoría se puede observar la manera en que las características propias del proyecto de innovación pueden modificar el nivel de confianza inicial debido a las proyecciones mentales que surgen al principio. Previo a la toma de decisiones se consideran el nivel de riesgo potencial, las expectativas, las predicciones sobre el futuro éxito del proyecto y su duración. Con estas consideraciones se generan hipótesis sobre la efectividad de la relación para el cumplimiento exitoso de los objetivos del proyecto o de la relación.

Como se mencionó, la innovación es un proceso social que implica formar y mantener relaciones intergrupales e interpersonales. La séptima categoría de antecedentes engloba ciertas particularidades a tomar en cuenta sobre el equipo de innovación, tales como: motivación por compensaciones, objetivos claros, estabilidad de los miembros del proyecto, integridad en el comportamiento y decisiones, compromiso, competencias y habilidades, comunicación - disciplina, enfoque de prueba y error-y flexibilidad. Dichos elementos son vitales para colaborar entre grupos.

Sin embargo, toda relación de grupo se reduce a relaciones interpersonales entre sus miembros y entre los miembros del equipo socio; por lo que los rasgos a nivel personal tienen injerencia en el nivel de confianza inicial de una relación. A continuación se enlistan algunos de estos atributos: adscripción organizacional; características profesionales y de personalidad; cualidades morales; amistades personales; preocupaciones de tipo social; nivel de educación; grado de competencia profesional; confiabilidad que transmiten; previsibilidad de sus acciones; estabilidad; identificación política o religiosa; comportamiento cooperativo; inclinación hacia los comportamientos de monitoreo; credibilidad y fiabilidad, genuinidad e identidad social, casta, relación familiar o relación socio-local. Dichos atributos pueden ser evaluados subconsciente e intuitivamente o de manera racional por parte del futuro socio o integrante del equipo de innovación para tomar la decisión sobre confiar o no.

Una vez que se toma la decisión de iniciar una relación de colaboración, comienza una etapa subsecuente en la que se desarrolla el proyecto de innovación con interacciones e intercambios constantes de conocimiento entre todos los involucrados. En el transcurso de la relación, los socios comparten un gran número de eventos que traen como consecuencia 
cambios constantes en los niveles de confianza. La confianza requiere de experiencias para construirse, mantenerse o aumentar; pero en cualquier momento puede desaparecer hasta el punto de finalizar una relación sin haber concluido el proyecto (Lewicki et al., 1998). A pesar de esta inestabilidad, se cree que a mayor interacción de los socios se llegan a conocer lo suficiente como para predecir su comportamiento y mitigar la sensación de incertidumbre en la relación. La finalidad es que no existan rupturas en la confianza durante la ejecución del proyecto y que la relación pueda alcanzar un estado de madurez al concluir de manera exitosa. El estado de madurez de la relación se fundamenta en la identificación, el entendimiento y la aceptación de los valores del socio (Dwiveldi et al. 2003). De esta manera, es posible que la relación continúe y que surjan oportunidades para nuevos proyectos.

El inicio y el final de un proyecto son momentos en los que el sujeto de estudio puede recordar su postura ante el socio. Por lo tanto, cuando se realiza un cuestionario o se entrevista a un informante, se puede considerar la temporalidad de la confianza especificando el inicio y el fin del proyecto como marco de referencia para distinguir el cambio entre los niveles de confianza. Dichos momentos resultan fáciles de recordar para el informante. Además, se puede aprovechar el acercamiento para cuestionar sobre las eventualidades que propiciaron dicho cambio con la finalidad de detectar las particularidades del contexto y del tipo de proyecto. Para profundizar en el entendimiento del fenómeno es primordial analizar dichas eventualidades, operacionalizar aquellos conceptos que aparecen como particulares del contexto, elegir las dimensiones de la confianza que serán observadas y especificar su nivel de análisis.

\section{Operacionalización y dimensiones de la confianza}

La operacionalización es un proceso en el que se identifican variables que permiten observar y medir o representar constructos teóricos de manera empírica (Batthyány et al., 2011). Con base en la revisión de la literatura y las escalas utilizadas para operacionalizar la confianza se identificaron cinco niveles de análisis: 1 . regional o del entorno; 2. interorganizacional; 3. entre equipos de innovación; 4. intraorganizacional; y 5. interpersonal (tabla 2). El nivel de confianza regional o del entorno abarca dimensiones desde la confianza interpersonal generalizada o la predisposición a confiar en la mayoría de la gente; hasta la confianza en el Gobierno e instituciones. Esta última dimensión considera la percepción de transparencia en los procesos y recursos del Gobierno y el ambiente empresarial. La confianza interorganizacional comprende desde la reputación, las capacidades, 
los recursos, el conocimiento profesional, la transparencia, hasta el grado de responsabilidad y oportunismo.

La confianza entre equipos de innovación se refiere a los grupos de personas dentro de un mismo proyecto que comparten directamente sus conocimientos e ideas para generar, en conjunto, resultados innovadores. Los equipos pueden o no pertenecer a la misma organización; no obstante, el proyecto los une como una misma entidad de colaboración que trabaja aportando diferentes capacidades o habilidades complementarias. Este nivel es comúnmente investigado desde la perspectiva de la administración, la ingeniería y la mercadotecnia.

La confianza intraorganizacional está basada en la percepción que tienen los empleados de sus directivos y de los responsables sobre su benevolencia, apoyo, crítica constructiva, honestidad, confiabilidad, integridad, centralidad de las decisiones, entre otros aspectos. Este nivel de análisis es particularmente importante cuando el empleado que per-

Tabla 2

Operacionalización de la confianza según el nivel de análisis

\begin{tabular}{ll}
\hline $\begin{array}{c}\text { Tipos de confianza según } \\
\text { el nivel de análisis }\end{array}$ & \multicolumn{1}{c}{ Operacionalización y dimensiones } \\
\hline Regional o del entorno & $\begin{array}{l}\text { Confianza interpersonal generalizada, reputación de la región, } \\
\text { ambiente de innovación, confianza y calidad del gobierno, } \\
\text { sistema legal y las instituciones. }\end{array}$ \\
\hline Interorganizacional & $\begin{array}{l}\text { Reputación, conocimiento profesional, competencia, buena } \\
\text { voluntad, intereses mutuos, experiencias previas de coopera- } \\
\text { ción, cumplimiento de promesas, confiabilidad, franqueza, } \\
\text { veracidad, capacidad, de gestión, transparencia, constancia y } \\
\text { perseverancia; entendimiento, benevolencia, valor de la pala- } \\
\text { bra, conocimiento mutuo de las debilidades, oportunismo y } \\
\text { responsabilidad. }\end{array}$ \\
\hline $\begin{array}{l}\text { Entre equipos de innova- } \\
\text { ción }\end{array}$ & $\begin{array}{l}\text { Compromisos, promesas, habilidad para compartir informa- } \\
\text { ción, dependencia, transparencia y competencia. }\end{array}$ \\
\hline Intraorganizacional & $\begin{array}{l}\text { Benevolencia, actitud hacia el trabajo, apoyo, amistad, acep- } \\
\text { tación de crítica constructiva, compromiso, poder, dependen- } \\
\text { cia, honestidad, confiabilidad, integridad y centralidad de las } \\
\text { decisiones. }\end{array}$ \\
\hline $\begin{array}{l}\text { Honestidad, apertura, integridad, predisposición a confiar, } \\
\text { expectativas, valores, actitudes, dominación; interés y preo- } \\
\text { cupación por los demás; comprensión, disposición a apoyar } \\
\text { y preocupación por los demás; ideologías, convicciones, } \\
\text { creencias, valor de la palabra, cumplimiento, constancia y } \\
\text { perseverancia. }\end{array}$ \\
\hline Interpersonal & \\
\hline
\end{tabular}

Fuente: Elaboración propia con base en el análisis de la literatura. 
tenece al equipo de innovación no tiene una relación de amistad o familiar con los fundadores de la organización; cuando ha tenido o le han comunicado sobre experiencias negativas en otros departamentos o proyectos de la organización; o cuando se siente alejado de los valores y la cultura de la organización a la que pertenece. La confianza intraorganizacional es semejante a la interpersonal; la única diferencia es que se da entre individuos que pertenecen a una misma organización, quienes ya han interiorizado o simplemente comparten los principios y valores de la cultura organizacional. Este nivel ha despertado interés en investigadores del campo de la administración, la mercadotecnia y la psicología social, debido al impacto que tiene la calidad de las relaciones de jerarquía y poder en el flujo de ideas e innovación, como se demuestra en el estudio realizado por Ruppel y Harrington (2000).

La confianza interpersonal se refiere a la relación que existe entre los representantes de diferentes organizaciones y se puede identificar por su enfoque en las características intrínsecas de cada individuo que no están relacionadas a la organización a la que pertenecen. Para operacionalizar la confianza interpersonal se seleccionan preguntas enfocadas en la percepción que se tiene sobre cada uno de los involucrados en el proyecto de innovación, independientemente de que pertenezcan o no a la misma organización. Se pueden considerar cuestiones enfocadas en el grado de honestidad, apertura, actitudes, dominación, disposición a apoyar, entre otras características que se perciben de los demás. El nivel interpersonal es estudiado principalmente con aproximaciones teóricas de carácter psicológico.

Las escalas de medición de los constructos, las hipótesis, las preguntas de investigación y el tipo de informantes se eligen según los niveles de análisis de la confianza y las dimensiones que sean pertinentes. Se debe mencionar que las escalas que se han diseñado, habitualmente atienden sólo algunas dimensiones de la confianza; sin embargo, han probado ser confiables desde el punto de vista estadístico, ya que reportan en todos los casos Alphas de Cronbach mayores a 0.70. No obstante, si se utilizara una metodología mixta se podrían anexar las dimensiones no consideradas en la escala para ampliar el entendimiento del fenómeno en un contexto en particular. Esto permitiría explicar con mayor profundidad el proceso de construcción de la confianza en relaciones que buscan generar innovación a través de la colaboración interorganizacional. 


\section{Propuesta de modelo para estudiar la confianza en proyectos de innovación}

Como resultado del análisis de la literatura seleccionada, se creó el siguiente proceso de construcción de la confianza para las relaciones de innovación colaborativa. El modelo propuesto tiene como objetivos: 1. Presentar gráficamente un modelo de flujo que permita identificar cada una de las etapas en la ejecución de un proyecto de innovación; 2. Identificar los momentos en los cuales la relación puede finalizar prematuramente debido a la falta de confianza; 3. Resaltar la importancia de la confianza inicial y final como momentos en los cuales se puede medir sus niveles para su posterior comparación; 4. Presentar la etapa de "desarrollo de proyecto" como un conjunto de eventualidades que pueden modificar continuamente el nivel de confianza entre los involucrados; 5 . Enfatizar que, una vez que termina una relación, se genera un acervo de experiencias y lecciones de aprendizaje que serán de utilidad para futuras colaboraciones; y 6 . Aclarar que, a pesar de que el modelo está inspirado en los niveles de análisis interorganizacional e interpersonal, este proceso puede adaptarse a otros niveles de confianza que involucren colaboraciones e intercambio de conocimientos.

El modelo de flujo (figura 1) sirve de guía para identificar los antecedentes y los constructos que se añadirán al estudio de la confianza según el contexto y posteriormente diseñar la metodología y los métodos de recolección de información adecuados. Éste abarca la temporalidad de un ciclo completo de un proyecto. Dicho ciclo puede verse interrumpido por eventualidades, las cuales pueden pausar o finalizar prematuramente el mismo. En caso de que lo anterior no ocurra, el ciclo puede repetirse al iniciar un nuevo proyecto entre los mismos actores. La decisión de colaborar de nuevo tiene su fundamento en el éxito relativo del proyecto anterior, el aprendizaje obtenido o en la experiencia positiva en la relación previa.

El grado de confianza inicial es la que permite la toma de decisiones y el nivel de formalidad de los acuerdos. Para medir el grado de confianza inicial se pueden utilizar escalas existentes ya probadas según los niveles de análisis que se quieran estudiar. Esto se puede realizar siempre y cuando se especifique que las preguntas buscan medir la confianza inicial; es decir, la confianza previa al inicio del proyecto de innovación. ${ }^{7}$ Es por esto que el modelo propuesto parte de los antecedentes que se mencionan a con-

\footnotetext{
Algunos ejemplos de escalas para medir la confianza, pueden encontrarse en los siguientes artículos: Benton y Maloni (2005, citado en Brinkhoff et al, 2015: 190); Brattström et al. (2015: 26); Costa y Anderson (2011; citado en Wilcox, 2016: 157); Peterson y Behfar (2003, citado en Hu et al., 2012: 792); Rempel et al. (1985, citado en Ruppel y Harrington, 2000: 321); y la escala de Williams y Du (2014: 984).
} 


\section{Figura 1}

Modelo del proceso de construcción de la confianza en proyectos de innovación

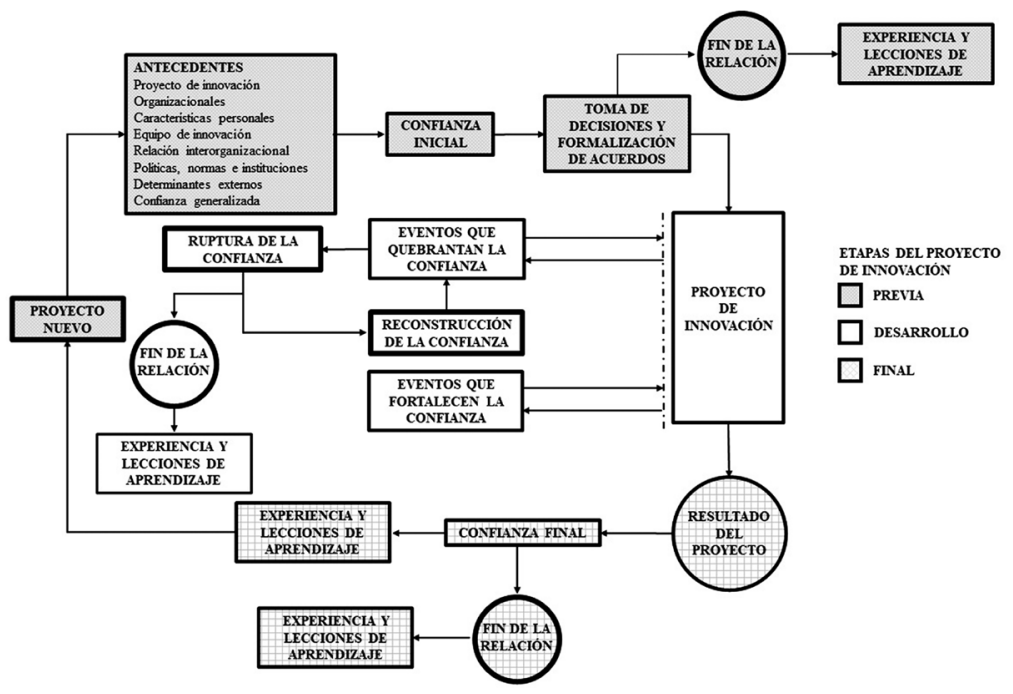

Fuente: Elaboración propia con base en la revisión de la literatura.

tinuación, los cuales influyen en la construcción de la confianza inicial: 1. El tipo de proyecto de innovación, 2. Las características organizacionales del futuro socio; 3. Las características personales de los representantes de cada organización, 4. El equipo de innovación, 5. La relación interorganizacional, 6. Las políticas, normas e instituciones, y 7. Los determinantes externos. El proyecto comienza una vez que los socios consideran que la confianza inicial es apta para llegar a un consenso y formalizar la relación; de lo contrario, el proyecto no se ejecuta.

La etapa de toma de decisiones y formalización de acuerdos es en la cual los involucrados comienzan un proceso de negociación. En dicha etapa se estipulan los objetivos, la duración del proyecto, la inversión, las responsabilidades y los responsables; la conformación del equipo de innovación, las fechas clave y entregables que corresponden a cada una de las partes; los planes a futuro; los riesgos e implicaciones; y otros aspectos de vital importancia para el proyecto. El hecho de decidir iniciar una relación con un socio implica que existe un grado, al menos mínimo, de confianza. No obstante, existen diversas herramientas que permiten fortalecer la confianza inicial para reducir el riesgo o formalizar la relación entre organizaciones al momento en que deciden colaborar.

En las relaciones interorganizacionales, las negociaciones usualmente van acompańadas de herramientas o acuerdos que protegen a los involucrados en 
caso de que se presente alguno de los riesgos previstos desde el inicio. A continuación se enlistan las herramientas más comunes (Hurley, 2012): 1. Entrega de prototipo como prueba de las capacidades de la organización; 2. Análisis en conjunto del riesgo; 3. Acuerdo para compartir el riesgo; 4. Acuerdo de riesgo por etapas; 5 . Empeño de recursos como seguro ante incumplimiento de acuerdos; 6 . Acuerdo para terminar la relación en caso de continuas pérdidas; 7 . Inspecciones por una en entidad independiente encargada del monitoreo o auditoría de recursos; 8. Contratación de pólizas de seguro, y 9. Concursos o convocatorias para recibir apoyo económico, los cuales permiten establecer los roles y responsabilidades mediante formatos establecidos. Este tipo de términos contractuales pueden ser vistos como meras formalidades o fortalecedores de la confianza interpersonal o interorganizacional. Sin embargo, se ha comprobado que sirven como herramientas para establecer la confianza inicial y permitir que ésta crezca conforme los intercambios continúen siendo beneficiosos.

Una vez que comienza el proyecto de innovación, ocurren eventualidades que fortalecen o transgreden la confianza. La desconfianza aparece cuando se rompen las expectativas o cuando se generalizan las expectativas debido a malas experiencias pasadas. Cuando las expectativas no se cumplen y la confianza se quebranta, se pueden producir reacciones, tales como: anomia, estado de shock, ansiedad, vergüenza, enojo o atribuciones negativas hacia el otro actor como locura o egoísmo (Zucker, 1985). Si acontece un evento que disminuya la confianza, se puede recurrir a un proceso de reconstrucción o inclusive acabar con la relación debido a la desconfianza. La desconfianza implica la atribución de una supuesta intencionalidad negativa por parte del socio. Por lo que la reconstrucción de la confianza se convierte en un proceso complejo, sobre todo cuando la comunicación entre los actores es limitada u obstaculizada.

Existen diversos tipos de eventualidades o actitudes de los socios, los cuales pueden quebrantar la confianza hasta el grado de dejar inconcluso un proyecto. Entre ellos se encuentran los siguientes: falta de consenso en los términos del proyecto, falta de comunicación o malos entendidos, objetivos no alineados, falta de flexibilidad, asimetría en las responsabilidades, poder y ganancias - maltrato, discriminación, menosprecio o burla-, poder y liderazgo centralizado, falsificación o copia de ideas o documentación, ruptura de palabra, falta de transparencia, actitudes sospechosas, rumores que afecten la reputación de alguno de los involucrados, falta a la confidencialidad, colaboración con la competencia, no honrar los contratos, renegociación o cambio en las condiciones del proyecto, indefinición de procesos, toma de decisiones importantes sin el consentimiento del socio, nuevos integrantes del equipo sin aviso previo, esconder información, mentiras, actos de corrupción, no cumplir 
objetivos o actividades en tiempo y forma, falta de definición de los roles de cada integrante, condicionamientos para la ejecución o cumplimiento de actividades, entre otros. A pesar de que muchas de estas eventualidades atenten contra el desarrollo o conclusión de un proyecto, existen diversas herramientas que versan desde el perdón, la comunicación directa, la supervisión por terceros hasta el replanteamiento de las condiciones del proyecto. Dichas herramientas pueden ser utilizadas para restablecer niveles sanos de confianza y lograr cumplir con los objetivos esperados.

El proceso de reconstrucción ocurre solamente cuando los involucrados consideran que vale la pena recuperar la relación. Por lo tanto, se toma la decisión de entrar a un proceso de negociación en el que se esclarecen los detalles de la falta y las verdaderas intenciones de quien la cometió para que de esta manera éste pueda ofrecer una disculpa y se le pueda otorgar el perdón (Van de Ven y Smith Ring, 2006). En el caso de que se reconstruya la confianza, el proyecto continúa bajo los mismos términos o se reestructuran los acuerdos, contratos o responsabilidades. De lo contrario, el proyecto o la relación finalizan, dejando como aprendizaje diversas experiencias y lecciones. Dichas experiencias y lecciones pueden incluir las siguientes: la especificidad de los contratos, los cobros con anticipación a clientes o inversionistas, los pagos a contra-entrega, la protección intelectual, la planeación y administración de los recursos, la supervisión externa, la anticipación a posibles problemáticas, los presupuestos considerando cambio en precios, las formas para prevenir rotación del personal, la especificidad de las cartas de confidencialidad, los criterios para la selección de proveedores, las vías de comunicación efectiva entre los integrantes del equipo, el tipo de liderazgo y seguimiento del proyecto para el cumplimiento de objetivos, la evaluación previa del conocimiento técnico del equipo y elección de responsabilidades, la consideración de las curvas de aprendizaje, los tiempos y medios de entrega de materiales para el proyecto, el cronograma realista para prevenir empalme de actividades consecutivas, los medios seguros para el almacenamiento de información y avances del proyecto, la documentación de procesos, entre otros aspectos.

Resulta evidente cómo el proceso de construcción de la confianza consume tiempo y recursos. A pesar de que en ocasiones puede llegar a ser un proceso tardado y costoso, permanecer en contacto continuo con los socios permite ahorrar tiempo en la búsqueda de información, favorece el intercambio constante de conocimiento y acelera el proceso de innovación. Los costos que permiten el aumento de confianza son económicamente irrecuperables de manera directa, pero son los que soportan un flujo continuo de innovación (Malecki, 2012). Como resultado, se espera que el equipo tenga un buen desempeño y que la innovación tenga mayores posibilidades de éxito. 
Al finalizar el proyecto, se puede medir el grado de confianza final entre los involucrados según el nivel de análisis elegido. Esta medición, arrojará información sobre el cambio en el nivel de confianza. Si éste resulta ser considerablemente distinto al nivel inicial, valdrá la pena adentrarse en las eventualidades que surgieron durante la ejecución del proyecto para identificar los detonadores del cambio. Esto permitirá observar las particularidades de la confianza en ese contexto de estudio o tipo de proyecto y de esta manera, comparar con otros contextos.

El modelo puede ser aplicado para proyectos intra o interorganizacionales, así como proyectos interpersonales de emprendedores que se asocian entre ellos para generar innovación. Dicho modelo representa la mayor aportación de este artículo para la literatura sobre la confianza. Como futura investigación se propone probar empíricamente a nivel regional el modelo teórico, así como las recomendaciones propuestas con la finalidad de abonar a la explicación sobre la confianza en contextos organizacionales y de emprendimiento.

\section{Conclusiones}

La innovación es un proceso social que depende principalmente de la colaboración de diferentes actores, quienes pueden participar en su desarrollo, implementación o comercialización. El entorno, los recursos, la cultura, el equipo de trabajo, la cooperación efectiva y la confianza son aspectos que determinan la generación de innovación. La confianza es la principal responsable de que se origine un ambiente apto para el intercambio de conocimiento, ideas y para que se disminuya la sensación de incertidumbre y riesgo en la toma de decisiones. En este trabajo se eligió utilizar la palabra confianza para referirse a cualquiera de sus niveles de análisis. De esta manera, la conceptualización de la confianza se expresa como sigue: una decisión que puede variar con el tiempo, está basada en expectativas sobre las intenciones o el comportamiento de los demás, funge como mediadora; y permite la vulnerabilidad y el riesgo en cualquier relación.

En la literatura analizada sobre la metodología, conceptualización y operacionalización utilizadas en el estudio de la confianza, se tienen cuatro hallazgos principales: en primer lugar, los constructos seleccionados son cualitativos pero gran parte de éstos son estudiados con metodologías puramente cuantitativas, que dejan de lado el aspecto social e interpretativo que poseen este tipo de relaciones. Esto representa un déficit en su explicación por lo cual se recomienda lo siguiente: 1. Identificar previamente los antecedentes y dimensiones a analizar, por medio de un acercamiento personal al contexto de estudio con entrevistas informales o 
etnografía; 2. Utilizar una metodología mixta en la que se incluyan entrevistas o etnografía para complementar la información sobre los antecedentes de la confianza y los eventos que tuvieron efecto sobre el nivel de confianza; 3 . Aplicar cuestionarios que permitan validar matemáticamente las relaciones; y 4. Triangular la información.

Un segundo hallazgo fue identificar que la confianza se considera como un concepto atemporal. En ese sentido, se propone que en la medición del nivel de confianza se consideren dos momentos clave de los proyectos: al inicio y al final. Una tercera cuestión se refiere a la importancia de preguntar sobre las eventualidades ocurridas durante el proyecto, ya que eso brinda información sobre los motivos por los cuales la confianza se fortalece o quebranta. Dicha información es de utilidad al momento de gestionar proyectos de innovación, así como para el establecimiento de normas y reglas.

En secciones anteriores se describieron las diferentes categorías de antecedentes, las cuales abarcan desde las características de la confianza generalizada hasta las singularidades de los integrantes del equipo. El cuarto hallazgo versa justamente sobre el efecto de éstos sobre la confianza inicial de la relación. Ese conocimiento previo se construye con base en referencias a terceros, medios de comunicación, cultura y experiencias previas, entre otros aspectos. La toma de decisiones y formalización de acuerdos es uno de los pasos subsecuentes dependiente de los antecedentes de la confianza. Es recomendable no perder de vista la importancia de esta cuarta cuestión y tomarla en cuenta al momento de elegir los constructos y de definir las preguntas de la escala.

Derivado de la revisión de literatura y las observaciones previas, se diseñó un modelo que emula el proceso de construcción de la confianza. El inicio del modelo comprende los antecedentes del socio así como del entorno en el que se suscribe el proyecto de innovación. Una vez establecidos los antecedentes se genera lo que se llama confianza inicial. Ésta puede ser medida mediante una escala común de confianza según el nivel de análisis que se quiera estudiar. Este nivel inicial permite la toma de decisiones y formalidad de acuerdos. En caso de que no se llegue a un consenso la relación termina, dejando en la memoria una serie de lecciones de aprendizaje y experiencia. En caso de formalizarse la relación, se comienza el proyecto de innovación. El modelo muestra claramente que el proceso de construcción de la confianza consume tiempo y recursos, requiere de experiencia, y está sujeto a altos grados de incertidumbre. Vale la pena tener esto muy presente, a efecto de no generar falsas expectativas en las estrategias pensadas para la generación de innovación.

Más particularmente, durante el proyecto de innovación ocurren eventualidades que fortalecen o transgreden la confianza. Si ocurre un evento que disminuya la confianza, se puede recurrir a un proceso de 
reconstrucción, el cual es costoso y complejo, o acabar con la relación por cuestiones de desconfianza. El proceso de reconstrucción ocurre solamente cuando los involucrados consideran que vale la pena recuperar la relación. Si se continúa el proyecto, se puede llegar a tener un resultado el cual pudiera desembocar en innovación. Al terminar el proyecto se puede medir la confianza final, la cual arrojará información sobre los cambios en el nivel de confianza. Esto permitirá observar las particularidades de la confianza en ese contexto de estudio y, de esta manera, comparar con otros contextos. En el centro de estas discusiones está la naturaleza humana de la confianza, como se encontró en este trabajo, y que hace aún más impredecible la generación de innovación: emociones y sentimientos como el miedo, la sospecha, la amistad, la esperanza y la desesperación.

Falta mucho por explicar y entender en este campo. La metodología mixta pudiera ser una manera de complementar el conocimiento que ya se tiene y continuar con el estudio de la confianza en sus diferentes contextos. El modelo y su propuesta teórico-metodológica pueden ser aplicados en diferentes tipos de proyectos: intra e interorganizacionales, interpersonales de emprendedores que se asocian para generar innovación, o en sistemas regionales de innovación para fomentar la colaboración. Se considera que el modelo propuesto en este artículo puede validarse mediante métodos cualitativos como la etnografía, y probarse empíricamente mediante modelos estadísticos de ecuaciones estructurales (SEM por sus siglas en inglés). Los modelos SEM son especialmente útiles en casos como el que planteamos, ya que las variables de interés son constructos, difíciles de medir por lo que se infieren de variables observables en las que se reflejan, comúnmente en las preguntas de las escalas probadas. Naturalmente su instrumentación requiere de un estudio de campo extenso entre responsables de proyectos de innovación colaborativa.

La innovación traspasa los límites de los procesos de investigación y desarrollo e involucra un sinfín de actores que pretenden abonar el desarrollo económico de su organización, región o país. Tanto por el lado de los antecedentes de la confianza, así como de su operacionalización y construcción, queda claro que la vertiente espacial proporciona un marco contextual de primer orden para la innovación colaborativa. La confianza generalizada del país o región en la que se realiza la investigación puede tener injerencia en el nivel de confianza inicial de cualquier relación debido a la cultura, las costumbres y las tradiciones relacionadas con este contexto. En ese sentido, la aplicación empírica de esta propuesta teóricometodológica a casos de estudio regionales, constituye una avenida de investigación futura muy promisoria. 


\section{Fuentes consultadas}

Ahuja, Gautam (2000), "Collaboration networks, structural holes, and innovation: a longitudinal study", Administrative Science Quarterly, 45 (3), Cornell University, Nueva York, Estados Unidos de América, pp. 425-455.

Antoine, Mélanie (2007), "Le succès des projets d'innovation interorganisationnels initiés dans le cadre d'une politique publique: réflexions et observations sur la nature de l'innovation et les modes de cooperation”, document de trabajo núm. 200711 (02), HEC - Ecole de Gestion de l'Université de Liège, Liège, Bélgica.

Arata, Adolfo; Raul Stegmaier, Christopher Nikulin y Francisco Soto (2011), "Identificación de factores críticos de innovación para modelo de medición de innovación por etapas que sean representativos para los indicadores internacionales de $\mathrm{I}+\mathrm{D}+\mathrm{i}$ mediante la formación de Cluster", Science for Innovación, 1 (0), Universidad Técnica Federico Santa María, Valparaíso, Chile, pp. 19-34.

Babiliute-Juceviciene, Rita; Giedrius Jucevicius y Kestutis Krisciunas (2013), "Trust development in inter-organizational relations of knowledge-intensive firms", Social Sciences, 78 (4), Kaunas, Lithuania, pp. 54-64, doi: 10.5755/j01.ss.78.4.3230.

Bastic, Majda y Gabrijela Leskovar-Spacapan (2006), "What do transition organizations lack to be innovative?", Kybernetes, 35 (7/8), University of Maribor, Maribor, Slovenia, pp. 972-992, doi: 10.1108/03684920610675030.

Batthyány, Karina; Mariana Cabrera, Lorena Alesina, Marianela Bertoni, Paola Mascheroni, Natalia Moreira, Florencia Picasso, Jessica Ramírez y Virginia Rojo (2011), Metodología de la investigación en ciencias sociales, Universidad de la República, Montevideo, Uruaguay.

Brattström, Anna; Hans Löfsten y Anders Richtnér (2015), "Similar, yet different: a comparative analysis of the role of trust in radical and incremental product innovation", International Journal of Innovation Management, 19 (4), Imperial College Press, Londres, Reino Unido, pp. 1-33, doi: 10.1142/S1363919615500437. 
Brinkhoff, Andreas; Özalp Özer y Gökçe Sargut (2015), "All you need is trust? An examination of inter-organizational supply chain projects", Production and Operations Management, 24 (2), Production and Operations Management Society, Miami, Estados Unidos de América, pp. 181-200, doi: 10.1111/poms.12234.

Boughanbouz, Chaker; Boualem Aliouat y Fatem Saci (2012), "Interorganizational trust in the competitiveness pole", ponencia presentada en The 11th World Congress of the IFSAM, University of Limerick, 12 de junio, Limerick, Irlanda.

Dwiveldi, Mridula; Raul Varman y Kaushal Saxena (2003), "Nature of trust in small firm clusters", The International Journal of Organizational Analysis, 11 (2), Information Age Publishing, Inc., Scottsdale, Estados Unidos de América, pp. 93-104.

Fang, Eric; Robert Palmatier, Lisa Scheer y Ning Li (2008), "Trust at different organizational”, Journal of Marketing, núm. 72, American Marketing Association, Chicago, Estados Unidos de América, pp. 80-98.

Fritsch, Michael y Viktor Slavtchev (2011), "Determinants of the efficiency of Regional Innovation Systems”, Regional Studies, 45 (7), Regional Studies Association, Brighton, Reino Unido, pp. 905918, doi: 10.1080/00343400802251494.

Gulati, Ranjay; Nitin Nohria y Akbar Zaheer (2000), "Strategic networks", Strategic Management Journal, 21 (3), John Wiley \& Sons, Nueva Jersey, Estados Unidos de América, pp. 203-215.

$\mathrm{Hu}$, Meng-Lei Monica; Tsung-Lin Ou, Haw-Jeng Chiou y Lee-Cheng Lin (2012), "Effects of social exchange and trust on knowledge sharing and service innovation", Social Behavior \& Personality: an International Journal, 40 (5), Society for Personality Research, Palmerson Norte, Nueva Zelanda, pp. 783-800, doi: 10.2224/ sbp.2012.40.5.783.

Hummels, Harry y Hans Rosendaal (2001), "Trust in scientific publishing", Journal of Business Ethics, 34 (2), Kluwer Academic Publishers, Dordrecht, Holanda, pp. 87-100, doi: 10.1023/A:1012282216211. 
Hurley, Robert (2012), The decision to trust: how leaders create high-trust organizations, Josey-Bass, San Francisco, Estados Unidos de América.

Hussein, Ashatu (2009), "The use of triangulation in social sciences research: can qualitative and quantitative methods be combined?", Journal of Comparative Social Work, 4 (1), pp. 1-12. <http:// journal.uia.no/index.php/JCSW/article/view/212>, 2 de enero de 2018.

Ingstrup, Mas Bruun (2013), "Facilitating different types of clusters", Management Revue, 24 (2), Rainer Hampp Verlag, Mering, Alemania, pp. 133-150, doi: 10.1688/1861-9908_mrev_2013_02_ Ingstrup.

Jirotka, Marina; Rob Procter, Mark Hartswood, Roger Slack, Andrew Simpson, Catelijne Coopmans, C., Chris Hinds, y Alex Voss (2005), "Collaboration and trust in healthcare innovation: The eDiaMoND case study", Computer Supported Cooperative Work (CSCW), 14 (4), Springer Science+Business Media, Inc., Berlín, Alemania, pp. 369-398, doi: 10.1007/s10606-005-9001-0.

Kern, Thomas y Leslie Willcocks (2002), "Exploring relationships in information technology outsourcing: the interaction approach", European Journal of Information Systems, 11 (1), Operational Research Society Ltd., Birmingham, Inglaterra, pp. 3-19, doi: $10.1057 /$ palgrave/ejis/3000415.

Lemus Delgado, Daniel Ricardo; Miguel Ángel Montoya Bayardo y Muricio Cervantes Zepeda (2015), "Los parques científicos tecnológicos como espacios para la innovación: evidencias del centro del software en Guadalajara”, Intersticios Sociales, núm. 9, El Colegio de Jalisco, Zapopan, Jalisco, México, p. 30.

Lewicki, Roy y Barbara Benedict Bunker (1995), “Trust in relationships: A model of development and decline", en Barbara Benedict Bunker, Jeffrey Z. Rubin et al., Conflict, cooperation, and justice: Essays inspired by the work of Morton Deutsch, John Wiley \& Sons, Nueva Jersey, Estados Unidos de América, pp. 133-173.

Lewicki, Roy; Daniel McAllister, y Robert Bies (1998), “Trust and Distrust: New Relationships and Realities”, Academy of Management 
Review, 23 (3), Academy of Management, Nueva York, Estados Unidos de América, pp. 438-458, doi: 10.2307/259288.

López-Jiménez, Liliana (2010), "Contracts, trust and information systems innovation”, Cuadernos. EBAPE.BR, 8 (2), FGV EGAPE, Río de Janeiro, Brasil, pp. 323-338.

Malecki, Edward (2012), "Regional social capital: why it matters", Regional Studies, 46 (8), Regional Studies Association, Brighton, Reino Unido, pp. 1023-1039, doi: 10.1080/00343404.2011.607806.

Maurer, Indre (2010), "How to build trust in inter-organizational projects: The impact of project staffing and project rewards on the formation of trust, knowledge acquisition and product innovation", International Journal of Project Management, 28 (7), Elsevier Ltd e IPMA, Amsterdam, Holanda, pp. 629-637, doi: 10.1016/j. ijproman.2009.11.006.

Molina-Morales, Xavier; Teresa Martínez-Fernández y Vanina Jasmine Torlò (2011), "The dark side of trust: the benefits, costs and optimal levels of trust for innovation performance", Long Range Planning, 44 (2), Elsevier Ltd, Amsterdam, Holanda, pp. 118133, doi: 10.1016/j.lrp.2011.01.001.

Nooteboom, Bart (2006), "Trust and innovation", en Reinhard Bachmann y Akbar Zaheer (eds.), Handbook of trust research. 1st., Edward Elgar Publishing Limited, Cheltenham, Reino Unido, pp. 106-122.

OECD (Organización para la Cooperación y Desarrollo Económico) Eurostat (2005), Oslo Manual: guidelines for collecting and interpreting innovation data, OECD Publishing (The Measurement of Scientific and Technological Activities), Paris, France, doi: 10.1787/9789264013100-en.

O'Mara, Margaret (2010), "Don't try this at home: You can't build a new Silicon Valley just anywhere", Foreign Policy, septiembre/octubre 2010, pp. 149-151, <https://foreignpolicy.com/2010/08/06/ dont-try-this-at-home/>, 5 de mayo de 2018.

Rampersad, Giselle; Pascale Quester y Indrit Troshani (2010), "Examining network factors: commitment, trust, coordination and harmony", Journal of Business \& Industrial Marketing, 25 (7), Emerald Group 
Publishing Limited, Bingley, Reino Unido, pp. 487-500, doi: 10.1108/08858621011077727.

Rousseau, Denise, Sim Sitkin, Ronald Burt, y Colin Camerer (1998), "Not so different after all: a cross-discipline view of trust", Academy of Management Review, 23 (3), Academy of Management, Nueva York, Estados Unidos de América, pp. 393-404.

Ruppel, Cynthia y Susan Harrington (2000), "The relationship of communication, ethical work climate, and trust to commitment and innovation", Journal of Business Ethics, 25 (4), Kluwer Academic Publishers, Dordrecht, Holanda, pp. 313-328, doi: 10.1023/A:1006290432594.

Salas, Claudia; Gabriela Susunaga e Ismael Aguilar-Barajas (2003), "The role of innovation in regional economic development: some lessons and experiences for policy making", en David Gibson; Chandler Stolp, Pedro Conceicao y Manuel Heitor (eds.), System and policies for the global learning economy, Preager Publisher Westport, Londres, Inglaterra, pp. 113-135.

Seppänen, Risto; Kirsimarja Blomqvist y Sanna Sundqvist (2007), “Measuring inter-organizational trust-a critical review of the empirical research in 1990-2003", Industrial Marketing Management, 36 (2), Elsevier Inc., Amsterdam, Holanda, pp. 249-265, doi: 10.1016/j.indmarman.2005.09.003.

Sie, Rory; Marlier Bitter-Rijpkema, Slavi Stoyanov y Peter Sloep (2014), "Factors that influence cooperation in networks for innovation and learning", Computers in Human Behavior, 37 (2014), Elsevier Ltd., Amsterdam, Holanda, pp. 377-384, doi: 10.1016/j.chb.2014.04.033.

Van de Ven, Andrew y Peter Smith Ring (2006), "Relying on trust in cooperative inter-organizational relationships", en Reinhard Bachmann y Akbar Zaheer (eds.), Handbook of trust research, Edward Elgar Publishing Limited, Cheltenham, Inglaterra, pp. 144-164.

Van Hemert, Patricia; Peter Nijkamp y Enno Masurel (2013), "From innovation to commercialization through networks and agglomerations: Analysis of sources of innovation, innovation capabilities and performance of Dutch SMEs", Annals of Regional Science, 50 (2), Amsterdam, Holanda, pp. 425-452, doi: 10.1007/s00168012-0509-1. 
Wei, Jiang; Haiyan Zhu y Yantao Cui (2006), “The trust mechanism between enterprises in traditional industrial clusters: some empirical evidence from Zhejiang area of China", IEEE International Conference on Management of Innovation and Technology, junio, Singapore, China, pp. 21-23.

Wei, Luan-Ying; Chung-Hsiung Fang y Guan-Li Chen (2008), “Trust relationships in inter-organizational learning alliance - using the bicycle industry's a-team as example", Proceedings of the 4th IEEE International Conference on Management of Innovation and Technology, ICMIT, septiembre, Bangkok, Tailandia, pp. 21-24.

Wilcox, Lynette (2016), The role of trust in university-industry research partnership performance. Virginia Polytechnic Institute and State University, Blascksburg, Estados Unidos de América, <https:// vtechworks.lib.vt.edu/bitstream/handle/10919/70927/Wilcox_ LF_D_2016.pdf?sequence $=1>, 5$ de enero de 2018 .

Williams, Christopher y Juana Du (2014), "The impact of trust and local learning on the innovative performance of MNE subsidiaries in China”, Asia Pacific Journal of Management, 31 (4), Springer, Nueva York, Estados Unidos de América, pp. 973-996, doi: 10.1007/s10490-014-9390-z.

WVS (World Values Survey) (2015), World Values Survey Wave 6 20102014 Official aggregate v.20150418, WVS, Estocolmo, Suecia, <http://www.worldvaluessurvey.org/WVSContents.jsp>, 5 de enero de 2018.

Zucker, Lynne (1985), "Production of trust: institutional sources of esconomic structure, 1840 to 1920 ", documento de trabajo núm. 85, Institute of Industrial Relations, Los Ángeles, Estados Unidos de América.

Recibido: 27 de febrero2018. Revisado: 11 de abril 2018. Aceptado: 7 de mayo 2018.

Gabriela Pino García. Doctoranda en Ciencias Sociales por el Tecnológico de Monterrey, campus Monterrey, México. Su investigación actual gira en torno a la confianza en proyectos de innovación colaborativa. 
Ismael Aguilar Barajas. Doctor en Planeación Urbana y Regional por la Escuela de Economía y Ciencia Política, Londres, Inglaterra. Profesor titular de Economía en la Escuela de Ciencias Sociales y Gobierno e Investigador Asociado en el Centro del Agua para América Latina y el Caribe, del Tecnológico de Monterrey, campus Monterrey, México. Es miembro del Sistema Nacional de Investigadores, nivel II. Su investigación y publicación giran en torno a la urbanización, a la gestión del agua urbana y de la región fronteriza México-Estados Unidos. Entre sus últimas publicaciones destacan: en coautoría, "Prólogo", en Ismael Aguilar-Barajas, Noé Arón Fuentes-Flores, Juan Antonio Le Clercq Silvia Nuñez-García, Lorena Ruano Gómez y Marta Tawil Kuri (coords.), Integración en América del Norte (1994-2016): Reflexiones desde el PIERAN, Colegio de México, Ciudad de México, México, pp. 11-16 (2017); en coautoría, Infectious Intestinal Diseases and Residential Water Services in Mexico: a Spatial Analysis, AIMS Press, Springfield, Estados Unidos de América (2017); "Agua, ciudades y desarrollo sostenible. El contexto”, en Ismael Aguilar-Barajas, Jürgen Mahlknecht; Jonathan Kaledin, Mariann Kjellén y Abel Mejía-Betancourt (eds.), Agua y ciudades en América Latina: Retos para el desarrollo sostenible, Routledge, Nueva York, Estados Unidos de América, pp. 13-31 (2015).

Edgardo Arturo Ayala Gaytán. Doctor en Estudios de Negocios por la Universidad de Tulane, Estados Unidos de América. Actualmente es profesor asociado de la Escuela Nacional de Ciencias Sociales del Tecnológico de Monterrey, campus Monterrey, México. Es miembro del Sistema Nacional de Investigadores, nivel II. Sus áreas de investigación son análisis sectorial y desarrollo económico, y dentro de este campo ha abordado problemáticas asociadas al estudio de impacto de reformas sectoriales, finanzas públicas y desarrollo regional. Entre sus últimas publicaciones destacan: en coautoría, Welfare effects of the Telecommunication Reform in Mexico, Elsevier, Nueva York, Estados Unidos de América (2018); en coautoría, La disposición a pagar más por productos vinculados a la RSE. Evidencia de un análisis conjunto en México, Universidad Nacional Autónoma de México, Ciudad de México, México (2018); Daily use of time, personal characteristics and experienced well-being, Emerald Publishing Limited, Bingley, Reino Unido (2017). 